\title{
Kinetic Control of the Supramolecular Chirality of Porphyrin J-Aggregates
}

\author{
Alessandro Sorrenti, ${ }^{[a, c]}$ Zoubir El-Hachemi, ${ }^{[a]}$ Oriol Arteaga, ${ }^{[b, d]}$ Adolf Canillas, ${ }^{[b]}$ \\ Joaquim Crusats, ${ }^{[a]}$ and Josep M. Ribo* ${ }^{[a]}$
}

\begin{abstract}
The aggregation of achiral sulfonatophenyl- and phenyl-meso-substituted diprotonated porphyrins to chiral J-aggregates is a hierarchical noncovalent polymerization process preceded by a critical nucleation stage. This allows significant enantiomeric excesses by the formation of a few primary nuclei and the control of their
\end{abstract}

growth by the effect that flows (imperfect mixing) have on the secondary nucleation of the J-aggregate particles. In addition, the results strongly suggest

Keywords: chirality • circular dichroism • porphyrinoids • self-assembly $\cdot$ supramolecular chemistry that when only one species of aggregate predominates, the $\mathrm{CD}$ signals of the three excitonic bands in the visible region (around 420, 490, and $700 \mathrm{~nm}$ ) show the same sign. Thus, differences on their relative sign would be due to the presence of different species.

\section{Introduction}

The formation of J-aggregates of amphiphilic diprotonated porphyrins is a hierarchical self-assembly process, which not only depends on the type of meso substituents at the macrocycle periphery, but also on the experimental parameters of their preparation. ${ }^{[1]}$ In our opinion, the structure of these $\mathrm{J}$ aggregates is not known, in spite of being of high interest, ${ }^{[2]}$ because of the formation of different species owing to yet unknown effects of the experimental parameters of preparation. These different species would differ structurally at the molecular level, but they would show very similar UV/Vis absorption spectra and rather similar morphologies at the nanoscale level. The results reported herein are in agreement with this assumption. The experimental procedure of preparation is little controlled, mostly because we tacitly assume that self-assembling systems are obtained under thermodynamic control. In this respect, the title J-aggregates are a paradigm of the complexity, arising from self-assembly and auto-organization processes. However, their study is fa-

[a] Dr. A. Sorrenti, Dr. Z. El-Hachemi, Dr. J. Crusats, Prof. J. M. Ribo Department of Organic Chemistry, Institute of Cosmos Science University of Barcelona (UB-IEEC)

c. Martí i Franquès 1, 08028 Barcelona, Catalonia (Spain)

E-mail: asorrenti@ub.edu jmribo@ub.edu

[b] Dr. O. Arteaga, Dr. A. Canillas

Department of Applied Physics, IN ${ }^{2} \mathrm{UB}$

University of Barcelona

c. Martí i Franquès 1, 08028 Barcelona, Catalonia (Spain)

[c] Dr. A. Sorrenti

Present address: Department of Chemistry, Faculty of Sciences Universitat Autònoma de Barcelona, 08193 Bellaterra, Catalonia (Spain)

[d] Dr. O. Arteaga

Present address: Department of Chemistry, New York University 31 Washington Place, New York, NY 1003 (USA) cilitated, compared with those of other systems, because of the unique properties of the porphyrin chromophore. ${ }^{[3]}$ Therefore, the existence of selection effects, acting on the bifurcation branch of the hierarchical self-assembly, as reported herein, is of general interest because it could be extrapolated to many other supramolecular systems. The results presented herein, on the chiro-optical properties of $\mathrm{J}$ aggregates of the porphyrins of Scheme 1, show the exis-

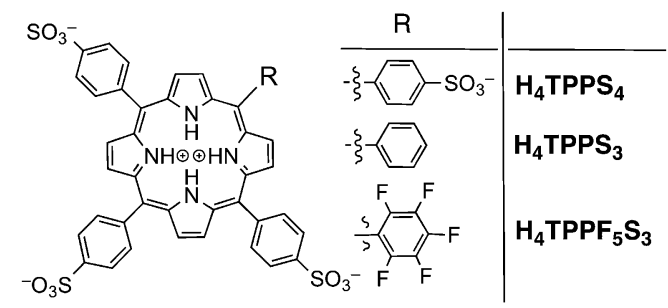

Scheme 1.

tence of a critical nucleation step that leads to chiral J-aggregates and that the control of the "primary-nucleation" step and of the growth of the first nuclei may lead to significant enantiomeric excesses. Therefore, the results show evident analogies with the spontaneous emergence of high enantiomeric excesses in the crystallization of achiral compounds $^{[4]}$ that yield racemic conglomerates. ${ }^{[5]}$

Recently, the existence of two different aggregation stages was reported for 5-pentafluorophenyl-10,15,20-tris(4-sulfonatophenyl)porphyrin $\left(\mathrm{H}_{4} \mathrm{TPPF}_{5} \mathrm{~S}_{3}\right){ }^{[1 \mathrm{~g}]}$ this may be extrapolated to the whole series of related porphyrin J-aggregates: 1) At high concentrations of J-aggregates, the behavior of the system is that characteristic of micellar and colloidal solutions, that is, attributable to effects on the electric double layer of the J-aggregate particles (e.g., flocculation). 2) The 
self-assembly of the monomer is driven exergonically by a cooperative noncovalent polymerization that starts from a critical nucleation stage. ${ }^{[1 \mathrm{~g}, 6]}$ In respect to the study of selfassembly and auto-organization phenomena in supramolecular systems, it is of general interest to study stage (2).

\section{Results and Discussion}

The study of the formation of $\mathrm{H}_{4} \mathrm{TPPF}_{5} \mathrm{~S}_{3} \mathrm{~J}$-aggregates (see Scheme 1) is more fruitful than the study of its phenyl and 4-sulfonatophenyl $\left(\mathrm{H}_{4} \mathrm{TPPS}_{3}\right)$ analogues because the polymerization is slower and less exergonic; this allows to follow the progress of the J-aggregate growth and even to detect some intermediate species. ${ }^{[1 \mathrm{~g}, 3]}$ Under experimental conditions that lead to low aggregation $\left(\mathrm{H}_{4} \mathrm{TPPF}_{5} \mathrm{~S}_{3} 10 \mu \mathrm{M}, \mathrm{HCl}\right.$ $0.1 \mathrm{M}$ ), a vigorous shaking of the flask leads to CD silent solutions of J-aggregates (Figure 1). However, when a free-
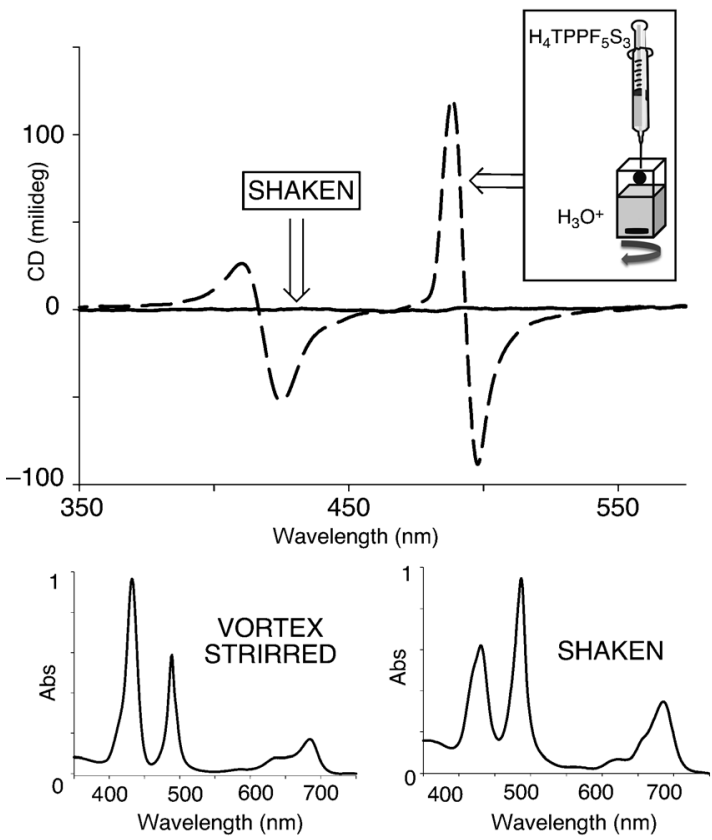

Figure 1. CD and UV/Vis spectra of $\mathrm{H}_{4} \mathrm{TPPF}_{5} \mathrm{~S}_{3}(10 \mu \mathrm{M}, \mathrm{HCl} 0.1 \mathrm{~m}) \mathrm{J}$-aggregates obtained under vigorous-shaking conditions ( $6 \mathrm{~h}$, full line) and in magnetically stirred conditions ( $24 \mathrm{~h}$, dashed line $)$.

base-porphyrin mother solution $(50 \mu \mathrm{L}, 0.5 \mathrm{~mm})$ was added to $\mathrm{HCl}(2.45 \mathrm{~mL}, 0.1 \mathrm{M})$ and magnetically stirred in a $1 \mathrm{~cm}$ square-section UV/Vis quartz cuvette, ${ }^{[7]}$ intense permanent $\mathrm{CD}$ bands were observed (Figure 1). The samples do not show linear dichroic contributions at $0^{\circ}$ and $45^{\circ}$ (inferred from the experimental Mueller matrix determined by 2-photomodulators generalized ellipsometry in transmission mode $)^{[8]}$ due to the small size and round shape of the J-aggregate particles $;^{[1 \mathrm{~g}]}$ hence, the circular optical polarization can be studied by a conventional CD spectrophotometer. Under neither of the experimental conditions, no differences could be detected in the UV/Vis spectra or in the particle shapes observed by AFM. Experiments in stagnant conditions are difficult to reproduce and lead to intermediate results between those of stirred and vigorous shaking conditions. Therefore, the results point to that the J-aggregates obtained under vigorous shaking correspond to a racemic mixture and that those obtained under vortex stirring correspond to a scalemic mixture. Under shaking conditions a relatively high number of chiral critical nuclei would be obtained, that is, a racemic mixture, whereas under vortex-stirring conditions, the formations of an Adam critical nucleus, or of a few critical nuclei, obviously give an initial enantiomeric excess (ee). Then, the subsequent selective growth of these first nuclei yields a detectable final ee of J-aggregates. Notice that this chiral effect is not related to the emergence of chirality by vortex stirring previously reported for elongated J-aggregate particles of $\mathrm{H}_{4} \mathrm{TPPS}_{3}$ (irreversible effect) ${ }^{[9]}$ or $\mathrm{H}_{4}$ TPPS $_{4}$ (reversible effect), ${ }^{[7,8]}$ which have been attributed to the mechanical deformation of the initially straight particles into chiral folded structures (see refs. [9-11]).

The CD signal obtained under vortex-stirring conditions was always of the same sign (see Figure 2), which, as previously reported, must be attributed to the inevitable presence of chiral biological debris in water and the effect it has on the chirality of these J-aggregates. ${ }^{[12]}$ This debris provides nucleation sites (heterogeneous primary nucleation mechanism) and, being chiral, it also selects the chirality sign of the primary nucleus. In ultra-pure water, the chiral cellular debris, or self-assembled structures of them, originate from the purification process of biological contamination by photolytic degradation and the size limit of the purification filters $(\geq 200 \mathrm{~nm})$. This is proven by the comparison to the chiral induction exerted by a molecular solute, such as enan-
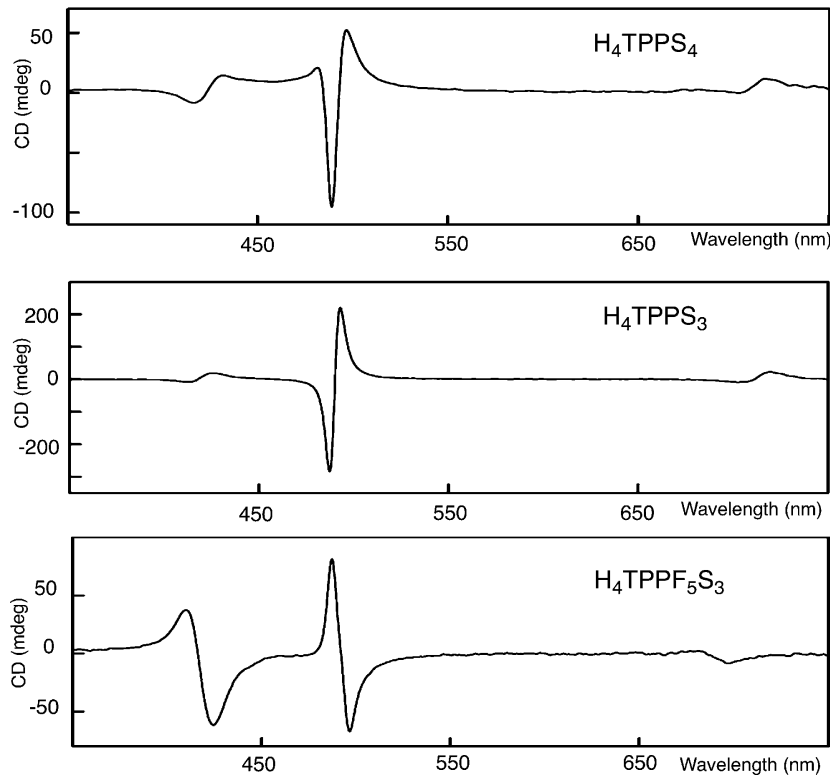

Figure 2. CD spectra showing chiral signs attributed to the chiral contamination present in water (see ref. [12]). $\mathrm{H}_{4}$ TPPS $_{4}(4 \mu \mathrm{M}, \mathrm{NaCl} 3 \mathrm{M}, \mathrm{HCl}$ pH 3); $\mathrm{H}_{4}$ TPPS $_{3}(13 \mu \mathrm{m}, \mathrm{HCl} \mathrm{pH} 3) ; \mathrm{H}_{4} \mathrm{TPPF}_{5} \mathrm{~S}_{3}(11 \mu \mathrm{m}, \mathrm{HCl} \mathrm{NaCl} 3 \mathrm{M}$, $\mathrm{pH} 1$; a lower $\mathrm{pH}$ value than for the other porphyrins is necessary because of its lower $\mathrm{p} K_{\mathrm{a}}$ value). 
tiopure tartaric acid. In this last case, the induction is not detected at concentrations below the $\mathrm{M}$ range (see Figure 3), which means that the chiral induction is actually exerted by the effect of a "chiral medium", and thus a high concentration of the solute is indeed necessary. In summary, the strong chiral induction exerted by the contaminant would occur in the heterogeneous nucleation step. Under strong shaking conditions, a high number of primary nuclei would be formed; this implies the participation of achiral nucleation sites (for example on the quartz walls), because the nucleation would surpass the number of the few available sites of the chiral contaminant; as a consequence, a racemic mixture of J-aggregates would be obtained.

Differences of the sign of the CD signals between J-aggregates of different porphyrins: The present results strongly suggest that the $C D$ spectra show bisignated signals of the same sign at the three excitonic absorption bands (ca. 420, 490 , and $700 \mathrm{~nm}$ ) of the aggregates. These three bands must be attributed to a J-aggregate species that shows excitons in different space directions, and not to different $\mathrm{H}$ - and $\mathrm{J}$-aggregates species. The 700 and the $490 \mathrm{~nm}$ bands correspond to the first and second excitonic singlets of the solid particle, respectively, and are both aligned in the same direction, and the $420 \mathrm{~nm}$ band corresponds to an exciton aligned orthogonally, or very near to $90^{\circ}$, to the former two excitons. This is supported by previous reports on the polarization of the emission spectra and confirmed by the measurement in the solution of the linear dichroism $\left(\operatorname{LD}\left(0^{\circ}\right)\right.$ and $\left.\mathrm{LD}^{\prime}\left(45^{\circ}\right)\right)$ of long-shaped particles at 420 and $490 \mathrm{~nm}$ and allows the determination of the chromophore vector orientation. However, the three absorptions show different ratios on their oscillator strengths depending on the monomer (for example $\mathrm{H}_{4} \mathrm{TPPF}_{5} \mathrm{~S}_{3}$ shows a more intense $420 \mathrm{~nm}$ band than its nonfluorinated counterparts) and on the hierarchical stage of the aggregation process. The $\mathrm{CD}$ signals show positive Cotton couplets for the J-aggregate of $\mathrm{H}_{4} \mathrm{TPPS}_{3}$, but negative Cotton couples for $\mathrm{H}_{4} \mathrm{TPPF}_{5} \mathrm{~S}_{3}$ (Figure 2). The chiral induction with $\mathrm{D}$ - and L-tartaric acid also leads to opposite signs for the CD bands when $\mathrm{H}_{4} \mathrm{TPPF}_{5} \mathrm{~S}_{3}$ is compared with $\mathrm{H}_{4} \mathrm{TPPS}_{4}$ and $\mathrm{H}_{4} \mathrm{TPPS}_{3}$ (Figure 3 ). The signs induced by the water contaminant in the three porphyrins correspond to those obtained with D-tartaric acid (Figure 3). The different sign in $\mathrm{H}_{4} \mathrm{TPPF}_{5} \mathrm{~S}_{3}$ compared with $\mathrm{H}_{4} \mathrm{TPPS}_{4}$ and $\mathrm{H}_{4} \mathrm{TPPS}_{3}$ should be attributed to strong supramolecular structural differences, rather than to a single physical effect of the pentafluorophenyl meso substituent, such as its partial dipole moment. In this respect, previous AFM results show that the J-aggregate particles of $\mathrm{H}_{4} \mathrm{TPPS}_{4}$ and $\mathrm{H}_{4} \mathrm{TPPS}_{3}$ form layered structures (multilayered and bilayered for $\mathrm{H}_{4} \mathrm{TPPS}_{4}{ }^{\left[{ }^{[b, 13]}\right.}$ and bilayered for $\mathrm{H}_{4} \mathrm{TPPS}_{3}{ }^{[0 b]}$ ), but $\mathrm{H}_{4} \mathrm{TPPF}_{5} \mathrm{~S}_{3}$ J-aggregates show monolayered structures. ${ }^{[1 \mathrm{~g}]}$

The chiral induction with the tartaric acid enantiomers is a competitive process with that of the chiral contaminant
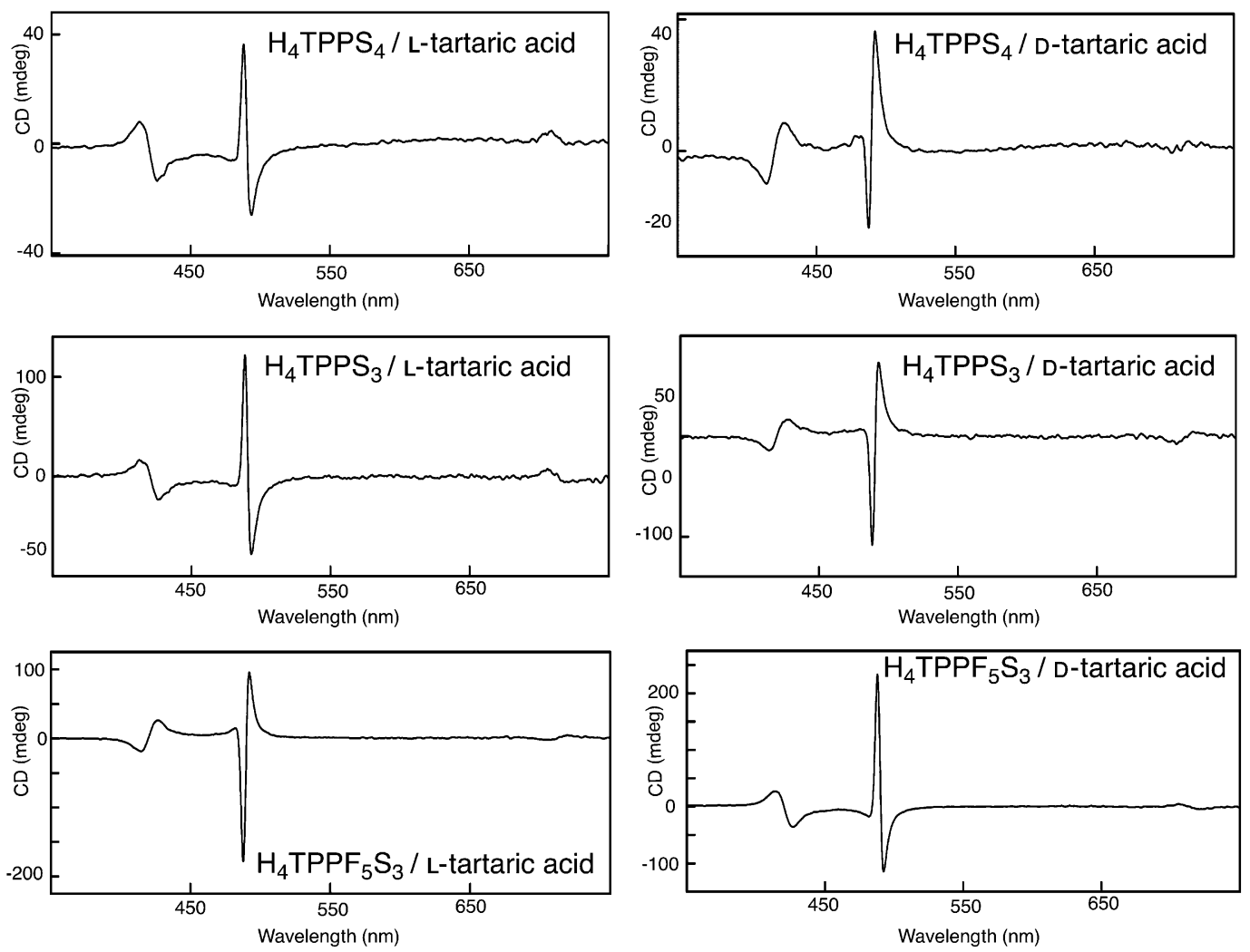

Figure 3. Characteristic CD spectra with signs originating from the effect of L- and D-tartaric acid $(0.4 \mathrm{M}), \mathrm{HCl}(0.1 \mathrm{M})$ on the $\mathrm{J}_{-}$-aggregates of $\mathrm{H}_{4} \mathrm{TPPS}_{4}$, $\mathrm{H}_{4} \mathrm{TPPS}_{3}$, and $\mathrm{H}_{4} \mathrm{TPPF}_{5} \mathrm{~S}_{3}(11 \mu \mathrm{M})$. 
present in water. This may explain why some differences in the final $\mathrm{CD}$ spectra are detected, depending on the preparation method of the J-aggregate solutions; for example addition of a mother neutral solution to an acidic solution, by diffusion in stagnant conditions, or in vortex-stirred conditions (see below). In the case of $\mathrm{H}_{4} \mathrm{TPPF}_{5} \mathrm{~S}_{3}$, the effect of the chiral water contaminant could only be overcome by the chiral tartaric acid when the mother solution of the freebase porphyrin, added to the acidic solution ( $\mathrm{HCl}$ plus tartaric acid), also contained tartaric acid salts.

The chiral-sign of the excitonic absorptions depend on the presence of different $\mathbf{J}$-aggregate species: The excitonic absorptions (at about 420, 490, and $700 \mathrm{~nm}$ ) of the aggregates generally show the same chiral sign (Figures 1 and 2). However, previous reports ${ }^{[14]}$ show that in some cases, the CD chiral sign of the $420 \mathrm{~nm}$ band can be of opposite sign compared with those of the 490 and $700 \mathrm{~nm}$ bands and that the $490 \mathrm{~nm}$ signal can show patterns different to that of a pure bisignate band. Generally, these irregular CD spectra are recorded at the first aggregation stages and eventually change to signals with bisignate patterns of the same sign for the three excitonic absorptions. Below we show examples on how different methods of fostering aggregation can lead to CD spectra that must be attributed to the simultaneous presence of more than one sort of aggregated species. All these facts imply that CD spectroscopy is more sensitive to the detection of structurally different mesomorphs than simple UV/Vis spectroscopy, but obviously the presence of significant ee is necessary.

In the case of $\mathrm{H}_{4} \mathrm{TPPS}_{3} \mathrm{~J}$-aggregates, similar $\mathrm{CD}$ patterns in all the three excitonic absorptions were obtained by using different preparation methods. However, different anisotropic patterns of the CD signals, mainly that at $490 \mathrm{~nm}$, could be a consequence of the presence of different J-aggregate species absorbing at very similar wavelengths.

Changes on the CD spectra due to the presence of different species can be clearly inferred in the case of $\mathrm{H}_{4} \mathrm{TPPF}_{5} \mathrm{~S}_{3}$ J-aggregates, because of its slower kinetics and the fact that, in contrast to $\mathrm{H}_{4}$ TPPS $_{4}$ and $\mathrm{H}_{4} \mathrm{TPPS}_{3}$, slight differences in the wavelengths of the absorption bands of some of the intermediate species can be detected by common UV/Vis spectrophotometry (wavelength differences $>1.5 \mathrm{~nm}$ ). ${ }^{[1 \mathrm{f}]} \mathrm{In}$ the case of $\mathrm{H}_{4} \mathrm{TPPF}_{5} \mathrm{~S}_{3}$, different preparation methods (e.g., the mixing order of the reactants) lead to $\mathrm{CD}$ spectra of opposite bisignate signs between the regions of approximately 420 and $490 \mathrm{~nm}$; in some cases the aggregates show different detectable absorption wavelengths around $490 \mathrm{~nm}$. The aggregation of $\mathrm{H}_{4} \mathrm{TPPF}_{5} \mathrm{~S}_{3}$, when controlled by laminar flows, leads to intense $\mathrm{CD}$ bands (vortex stirring in square section cuvettes, see ref. $[1 \mathrm{~g}]$ ). By following this procedure, and by using higher porphyrin concentrations and longer reaction times than those of ref. $[1 \mathrm{~g}]$, the shape of the CD spectra can only be interpreted as the superposition of CD couplets of different species (Figure 4). Note that the experiments of Figure 4 on $\mathrm{H}_{4} \mathrm{TPPF}_{5} \mathrm{~S}_{3}$ correspond to samples at an advanced stage of the hierarchical self-assembly. The recently
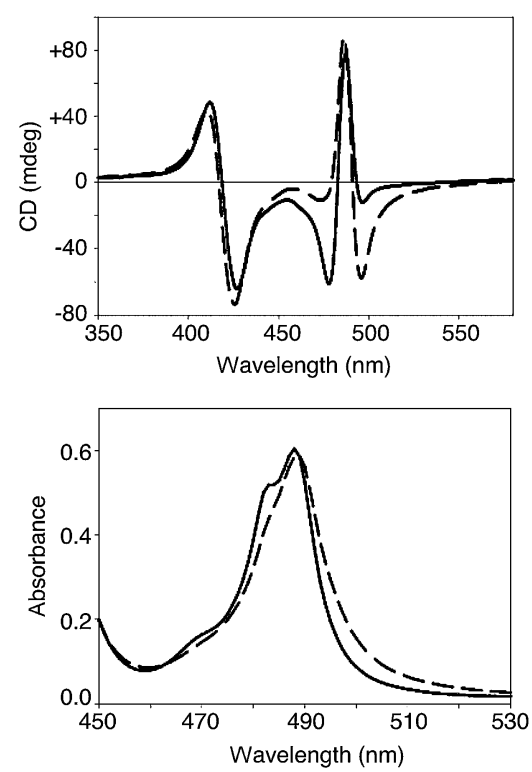

Figure 4. CD spectra (top) and UV/Vis absorption spectra (bottom) of two samples (continuous and dashed line) which contain different J-aggregate species at different concentrations. Samples were prepared by adding the necessary volume of a $\mathrm{H}_{4} \mathrm{TPPF}_{5} \mathrm{~S}_{3}$ stock solution $(0.5 \mathrm{~mm})$ to the proper amount of Milli-Q water, followed by the addition of the proper amount of $\mathrm{HCl}\left(1 \mathrm{M}\right.$, final concentrations: $\left[\mathrm{H}_{4} \mathrm{TPPF}_{5} \mathrm{~S}_{3}\right]=12 \mu \mathrm{M}$; [HCl] $0.1 \mathrm{M})$. The aggregation was conducted under magnetic stirring (vortex stirring) in a $1 \mathrm{~cm}$ quartz cuvette. The different absorption and $\mathrm{CD}$ spectra are due to different J-aggregate species as determined by the low reproducibility of the primary nucleation step (see ref. [1f]).

reported ${ }^{[1 \mathrm{~g}]}$ redshifted Soret bands corresponding to "oligomers" at 470 and $482 \mathrm{~nm}$ are not present in this sample, at least not in a significant concentration, but the presence of different species absorbing around $490 \mathrm{~nm}$ (488 and $492 \mathrm{~nm})^{[1 \mathrm{~g}]}$ are clearly detected. Furthermore, the different patterns of the CD signal around $490 \mathrm{~nm}$ can be correlated to two different absorptions in the UV/Vis spectra. It is very probable that different species show different rotational strengths and perhaps different $e e$ values; hence, inversion effects between the bands at 420 and $490 \mathrm{~nm}$ would occur during the progress of the hierarchical aggregation.

Similar effects on the CD spectra are also detected for $\mathrm{H}_{4}$ TPPS $_{4}$ (for examples, see ref. [14]), but the absorptions of the different aggregated species probably differ less than 1$0.5 \mathrm{~nm}$ and, therefore, are difficult to be experimentally detected by UV/Vis spectroscopy. Furthermore, the wellknown evolution with time of the anisotropy of the band at $490 \mathrm{~nm}$ may also be attributed to the change of the relative concentrations of J-aggregate species in the self-organization process.

It is reasonable to assume that the existence of homochiral-like species, at a structural small-size scale, showing CD signals of opposite CD signs at the excitonic absorptions, is a consequence of strong structural supramolecular differences, for example initial monolayered structures compared with bilayered and multilayered mature J-aggregates. In this respect, Figure 5 shows how the initial "oligomers" of 

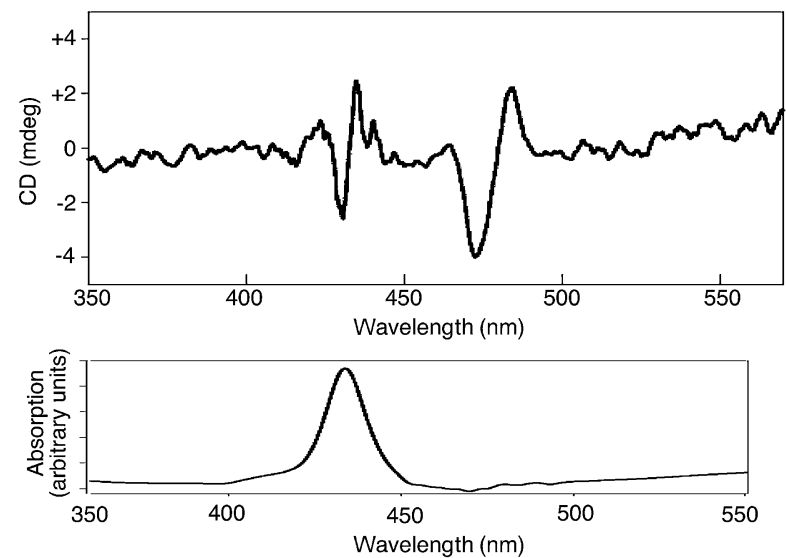

Figure 5. CD spectrum (inferred from 2-photomodulators generalized ellipsometry) and single beam absorption recorded at the very first aggregation stages ${ }^{[\mathrm{gg}]}$ of $\mathrm{H}_{4} \mathrm{TPPF}_{5} \mathrm{~S}_{3}(0.5 \mathrm{~mm})$ under magnetically stirred conditions (experimental conditions are the same as those in Figure 4: the small signal at the monomer absorption $(433 \mathrm{~nm})$ is an artifact due to depolarization effects (detected in the Mueller matrix measurement)). The absorbance value at the $482 \mathrm{~nm}$ band was around 0.01 (measurement with J\&M TIDAS spectrophotometer). The final mature J-aggregates of this sample showed negative Cotton couples at 419 and $488 \mathrm{~nm}$.

$\mathrm{H}_{4} \mathrm{TPPF}_{5} \mathrm{~S}_{3}$, absorbing around $480 \mathrm{~nm},{ }^{[1 \mathrm{~g}]}$ show opposite CD signs compared with those of the final aggregates.

Similar effects to those reported in the previous section were obtained by varying the acidity of the solutions. In the same experimental conditions as for the sample shown in Figure 1, but with acetic acid $(0.1 \mathrm{M}, \mathrm{pH} \cong 2.8)$ instead of $\mathrm{HCl}$ $(0.1 \mathrm{~m}, \mathrm{pH} \cong 1)$, spectra as that shown in Figure 6 were obtained, that is, with a signal at around $490 \mathrm{~nm}$ of opposite sign (compare with Figure 2), induced by the water contaminant. Figure 7 shows that for $\mathrm{H}_{4} \mathrm{TPPF}_{5} \mathrm{~S}_{3}$ with $1.7 \mathrm{~mm} \mathrm{HCl}$ $(\mathrm{pH} \cong 2.8)$, in contrast to the experiments with $0.1 \mathrm{M} \mathrm{HCl}$, different species of J-aggregates, showing different CD spectra, are obtained. The experiment illustrated in Figure 7 shows

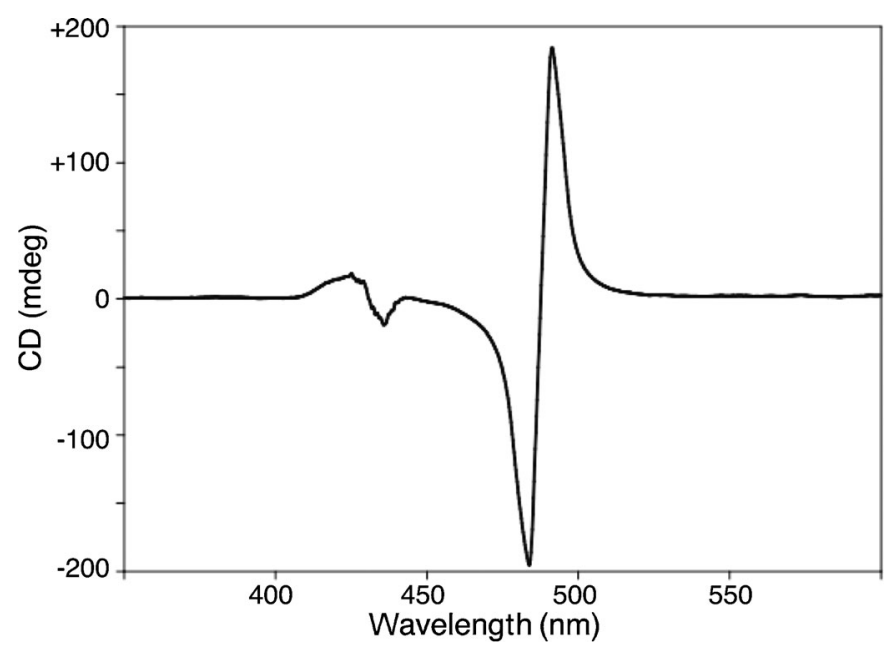

Figure 6. $\mathrm{H}_{4} \mathrm{TPPF}_{5} \mathrm{~S}_{3} \mathrm{~J}$-aggregates $\mathrm{CD}$ spectra obtained in water solutions $\left(10 \mu \mathrm{M} \mathrm{H}_{4} \mathrm{TPPF}_{5} \mathrm{~S}_{3}\right.$ and $0.1 \mathrm{M}$ acetic acid) by magnetic stirring in a squaresection UV/Vis cuvette. The CD sign of the redshifted Soret band is of opposite sign compared with that obtained in $\mathrm{HCl}(0.1 \mathrm{M}$, see Figure 2). that after the physical separation of different J-aggregate species (species of large size are with time deposited onto the quartz walls, whereas smaller ones remain in solution; Figure 7b), different CD spectra are obtained. Such acidity effects can be originated by the $\mathrm{pH}$ value, but also by counterion effects. In respect to a $\mathrm{pH}$ effect, note that $\mathrm{H}_{4} \mathrm{TPPF}_{5} \mathrm{~S}_{3}$ shows a significant $\mathrm{p} K_{\mathrm{a}}$-value difference compared with $\mathrm{H}_{4} \mathrm{TPPS}_{3}$ and $\mathrm{H}_{4} \mathrm{TPPS}_{4}\left(\mathrm{H}_{4} \mathrm{TPPF}_{5} \mathrm{~S}_{3} 3.8, \mathrm{H}_{4} \mathrm{TPPS}_{3}\right.$ 4.8 and $\mathrm{H}_{4} \mathrm{TPPS}_{4} 4.9$ ).

Preliminary results show that the signs of the CD spectra induced by tartaric acid also depend on the acidity of the solution (tartaric acid compared with tartaric acid plus $\mathrm{HCl}$ ) for both $\mathrm{H}_{4} \mathrm{TPPF}_{5} \mathrm{~S}_{3}$ and $\mathrm{H}_{4} \mathrm{TPPS}_{4}$, which would point both to $\mathrm{pH}$ as well as to a counterion effect.

The dependence of the order of mixing of the reactants, which yields different J-aggregates species (for a previous example, see ref. [1d] and references therein), can also be related to an effect of the $\mathrm{pH}$ value, because protonation would occur at specific sites of the porphyrin substrate at the initial stages of the self-assembly process and this would be dependent on the reactant mixing order and on the type of mixing. Such an effect is related to the actual proton concentration at the aggregation site at specific stages of the fast, initial, noncovalent polymerization. In this respect, the cooperative mechanism of proton diffusion in aqueous solution $^{[15]}$ causes a much faster diffusion of protons than those of the monomeric porphyrin, the corresponding counter anion, and the growing supramolecular species. In addition, when these supramolecular species are aligned in laminar flows, they show restriction on their lateral diffusion, which selectively inhibits cluster-to-cluster polymerization mechanisms. ${ }^{[\mathrm{g}]}$ Therefore, in the case of different substitution at the meso positions (i.e., for $\mathrm{H}_{4} \mathrm{TPPF}_{5} \mathrm{~S}_{3}$ and $\mathrm{H}_{4} \mathrm{TPPS}_{3}$ ) diastereoselective flow effects should also be assumed.

\section{Conclusion}

In the crystallization of achiral compounds, yielding enantiopure crystals as the more stable mesomorph (racemic conglomerates), deviations from the racemic outcome can be obtained by the chirality transfer of a first $e e$ of the primary nucleation and the subsequent production of more crystals by the secondary nucleation stage. Because the secondary nucleation is much faster than the primary nucleation process, the selective growth of the first nuclei is possible by two different ways: 1) slow growth in stagnant, unperturbed, low supersaturate solutions first described by Havinga; ${ }^{[16]}$ and 2) formation of new sites for secondary nucleation by eroding the crystals under vigorous stirring (Kondepudi experiments). ${ }^{[4]}$ The results reported herein does not establish which of these two mechanisms is at work, because the secondary nucleation mechanisms are slower compared with crystallizations. However, the results suggest that, in the hierarchical self-assembly of supramolecular systems undergoing spontaneous mirror symmetry breaking, the control of enantioselective growth would be sensitive to the experi- 

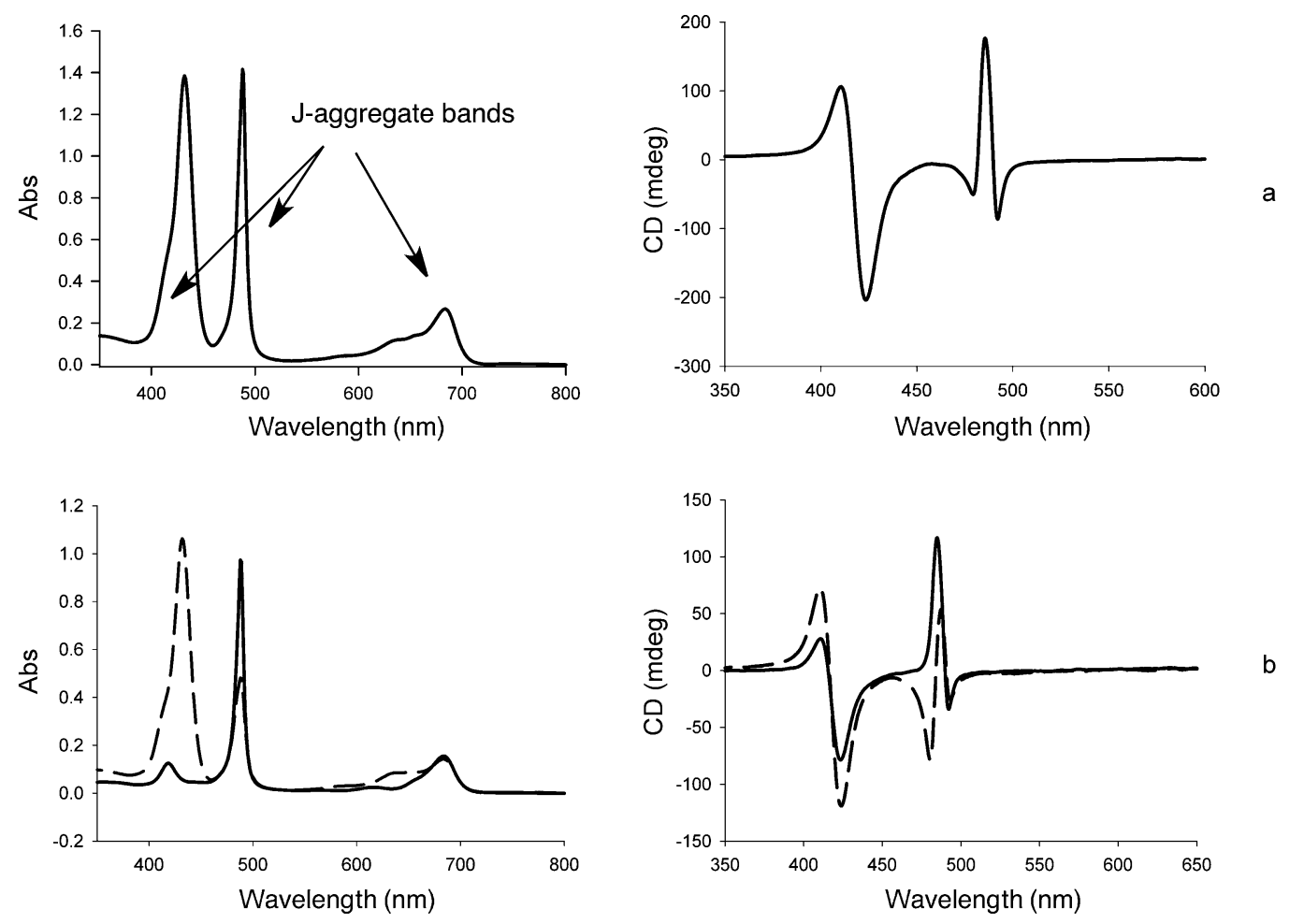

Figure 7. $\mathrm{CD}$ and $\mathrm{UV} / \mathrm{V}$ is spectra of $\mathrm{H}_{4} \mathrm{TPPF}_{5} \mathrm{~S}_{3} \mathrm{~J}$-aggregates obtained in water solutions $\left(10 \mu \mathrm{M} \mathrm{H}_{4} \mathrm{TPPF}_{5} \mathrm{~S}_{3}, 1.7 \mathrm{~mm} \mathrm{HCl}(\mathrm{pH} \cong 2.8)\right.$ and $\left.0.05 \mathrm{M} \mathrm{NaCl}\right)$ after 7 days with magnetic stirring in a square section cuvette. a) Whole solution. b) Empty cuvette (full line; J-aggregates absorbed at the cuvette walls) and separated solution (dashed line; J-aggregates in the solution).

mental parameters of reactants mixing and flow stirring and, therefore, fine tuning of these reaction conditions may be decisive for high ee values.

In addition, the results strongly suggest that the $\mathrm{CD}$ signals of the three aggregate excitonic absorptions in the visible region are all of the same sign and that samples with different signs would be a consequence of the presence of a mixture of structurally different, supramolecular J-aggregates.

\section{Experimental Section}

5,10,15,20-Tetrakis(4-sulfonatophenyl)-21H,23H-porphine $\left(\mathrm{H}_{2} \mathrm{TPPS}_{4}\right)$ and 5-phenyl-10,15,20-tris(4-sulfonatophenyl)-21H,23H-porphine $\left(\mathrm{H}_{2} \mathrm{TPPS}_{3}\right)$ were synthesized and purified as previously described. ${ }^{[17]} 5$-Pentafluorophenyl-10,15,20-tris(4-sulfonatophenyl)-21H,23H-porphine $\left(\mathrm{H}_{2} \mathrm{TPPF}_{5} \mathrm{~S}_{3}\right)$ was also synthesized as previously described. ${ }^{[1]]}$ Spectroscopic data and purity were as reported in the original publication.

Chiro-optical assessment: In spite of the fact that all the CD spectra reported in the figures herein were recorded with a Jasco 810 instrument, they did not differ significantly from those obtained by the determination of the full Mueller matrix by using 2-photomodulators generalized ellipsometry in transmission mode ${ }^{[8 a, 18]}$ This is because the linear polarizations are very low, even undetectable, in agreement with previous reports on the small size and rounded shape of the J-aggregate particles described herein. ${ }^{[19]}$

In solutions of long-shaped particles of $\mathrm{H}_{4} \mathrm{TPPF}_{5} \mathrm{~S}_{3}$ and $\mathrm{H}_{4} \mathrm{TPPS}_{4} \mathrm{~J}$-aggregates, the linear dichroic contribution at $0^{\circ}$ and $45^{\circ}$ were inferred from the experimental Mueller matrix J-aggregates. Particle alignment in the solution was induced by weak convection flows and by cuvette stirring. ${ }^{[7]}$ Measurements at the center of the cuvette show that the chromophore orientation of the 420 and the $490 \mathrm{~nm}$ absorptions are orthogonal $\left( \pm 5^{\circ}\right)$ and that the $490 \mathrm{~nm}$ is oriented along the long axis of the long-shaped particle.

AFM: Routine measurements in these samples were performed as previously reported. ${ }^{[19]}$

$\mathbf{p} \boldsymbol{K}_{\mathrm{a}}$ value of $\mathbf{H}_{2} \mathrm{TPPF}_{5} \mathbf{S}_{3}$ : The $\mathrm{pK}_{\mathrm{a}}$ value of $\mathrm{H}_{2} \mathrm{TPPF}_{5} \mathrm{~S}_{3}$ was determined by a spectroscopic method (see below), monitoring the absorbance change at $412\left(\mathrm{H}_{2} \mathrm{TPPF}_{5} \mathrm{~S}_{3}\right)$ and $432 \mathrm{~nm}\left(\mathrm{H}_{4} \mathrm{TPPF}_{5} \mathrm{~S}_{3}\right)$ of a solution of $\mathrm{H}_{2} \mathrm{TPPF}_{5} \mathrm{~S}_{3}$ or $\mathrm{H}_{4} \mathrm{TPPF}_{5} \mathrm{~S}_{3}\left(3.610^{-6} \mathrm{M}\right)$ at different $\mathrm{pH}$ values $(\mathrm{AcOH} /$ AcONa buffers at a total concentration $0.1 \mathrm{~m}$; absorbance measured at the same wavelength).

$$
\mathrm{p} K_{\mathrm{a}}=\mathrm{pH}+\log \frac{\left[\mathrm{H}_{4} \text { porph }\right]}{\left[\mathrm{H}_{2} \text { porph }\right]} \quad \frac{\left[\mathrm{H}_{4} \text { porph }\right]}{\left[\mathrm{H}_{2} \text { porph }\right]}=\frac{\mathrm{Abs}_{\mathrm{H}_{2} \text { porph }}-\mathrm{Abs}}{\mathrm{Abs}-\mathrm{Abs}_{\mathrm{H}_{4} \text { porph }}}
$$

Graphic interpolations $\left(412\right.$ and $432 \mathrm{~nm}$ ) yield a $\mathrm{p} K_{\mathrm{a}}=3.79 \pm 0.04$. The same method for $\mathrm{H}_{2} \mathrm{TPPS}_{3}$ and $\mathrm{H}_{2} \mathrm{TPPS}_{4}$ yielded no significantly different values $(4.8 \text { and } 4.9 \text {, respectively })^{[19]}$ for neither porphyrin. Previous reports ${ }^{[20]}$ give slightly lower values for $\mathrm{H}_{2} \mathrm{TPPS}_{4}$ : 4.6 and 4.8.

Preparation of J-aggregates: Water used in all experiments was obtained by Milli- $Q^{\circledast}$ Ultrapure water purification. The vortex-stirred sample shown in Figure 1 was prepared by adding a free-base-porphyrin mother solution $(50 \mu \mathrm{L}, 0.5 \mathrm{~mm})$ to $\mathrm{HCl}(2.45 \mathrm{~mL}, 0.1 \mathrm{~m})$ placed in a square-section UV/Vis cuvette. In the case of vigorous shaking the addition of the mother solution was followed immediately by very strong shaking of the closed UV/Vis cuvette. The final concentrations are those indicate in the caption of Figure 1. The samples shown in Figure 2, were prepared by adding $\mathrm{HCl}(2 \mathrm{M})$ to the porphyrin $/ \mathrm{NaCl}(3 \mathrm{M})$ solution. The final concentrations are those indicate in the caption of Figure 2. In all experiments performed herein, as well as those described in many previous reports, the sign of the $\mathrm{CD}$ couplets was the same as those show in Figure 2 (see ref. [6] for the effect of the chiral contaminant in water). The samples 
shown in Figure 3, were prepared by adding the corresponding small volume of a free-base-porphyrin mother solution (ca. $1.2 \mathrm{~mm}$ ) under magnetic stirring to $\mathrm{HCl}(0.1 \mathrm{M}, 2.5 \mathrm{~mL}$, final volume $2.5 \mathrm{~mL})$ and tartaric acid $(0.4 \mathrm{M})$ water solution placed in a square-section UV/Vis cuvette. In the case of $\mathrm{H}_{4} \mathrm{TPPF}_{5} \mathrm{~S}_{3}$, the expected change of signs by effect of L- or Dtartaric acid could only be obtained when the neutral porphyrin mother solution contained tartaric acid disodium salt; this mother solution was prepared by dissolving the porphyrin in a saturated solution (room temperature) of sodium L- or D-tartaric salts.

\section{Acknowledgements}

The study was financed by the Spanish Government (AYA2009-13920C02-01-02) and forms part of the COST Action CM0703. AS thanks the MICINN of the Spanish Government for the grant SB2009-0206. We thank Anna Miton for the $\mathrm{p} K_{\mathrm{a}}$ measurements on $\mathrm{H}_{2} \mathrm{TPPF}_{5} \mathrm{~S}_{3}$.

[1] a) R. F. Pasternack, C. Fleming, S. Herring, P. J. Collings, J. dePaula, G. deCastro, E. J. Gibbs, Biophys. J. 2000, 79, 550-560; b) A. S. R. Koti, J. Taneja, N. Periasamy, Chem. Phys. Lett. 2003, 375, 171-176; c) M. A. Castriciano, A. Romeo, V. Villari, N. Micali, L. M. Scolaro, J. Phys. Chem. B 2003, 107, 8765-8771; d) M. de Napoli, S. Nardis, R. Paolese, M. G. H. Vicente, R. Purrello, J. Am. Chem. Soc. 2004, 126, 5934-5935; e) C. Escudero, A. D'Urso, R. Lauceri, C. Bonaccorso, D. Sciotto, S. Di Bella, Z. El-Hachemi, J. Crusats, J. M. Ribo, R. Purrello, J. Porphyrins Phthalocyanines 2010, 14, 708-712; f) M. A. Castriciano, A. Romeo, G. De Luca, V. Villari, L. M. Scolaro, N. Micali, J. Am. Chem. Soc. 2011, 133, 765-767; g) A. Sorrenti, Z. El-Hachemi, J. Crusats, J. M. Ribo, Chem. Commun. 2011, 47, $8551-8553$.

[2] a) T. S. Balaban, Acc. Chem. Res. 2005, 38, 612-623; b) F. Würthner, T. E. Kaiser, C. R. Saha-Möller, Angew. Chem. 2011, 123, 34363473; Angew. Chem. Int. Ed. 2011, 50, 3376-3410.

[3] The porphyrin chromophore shows huge oscillator and rotational strengths and high-energy shifts between the monomer and the aggregate species. The progress of aggregation in these systems is monitored through the absorption bands of the monomer and $\mathrm{J}$-aggregate species (see, for example, ref. [1])

[4] a) D. K. Kondepudi, R. J. Kaufman, N. Singh, Science 1990, 250 , 975-976; b) J. M. McBride, R. L. Carter, Angew. Chem. 1991, 103, 298-300; Angew. Chem. Int. Ed. Engl. 1991, 30, 293-295; c) T. Buhse, D. Durand, D. Kondepudi, J. Laudadio, S. Spiker, Phys. Rev. Lett. 2000, 84, 4405-4408; d) D. K. Kondepudi, K. Asakura, Acc. Chem. Res. 2001, 34, 946-954.
[5] J. Jacques, A. Collet, S. H. Wilen, Enantiomers, Racemates, and Resolutions, Wiley, New York, 1981, pp. 32-88.

[6] T. F. A. De Greef, M. M. J. Smulders, M. Wolffs, A. P. H. J. Schenning, R. P. Sijbesma, E. W. Meijer, Chem. Rev. 2009, 109, $5687-$ 5754.

[7] For the effect of flows depending on the flask shape, see ref. [1f] and: O. Arteaga, A. Canillas, J. Crusats, Z. El-Hachemi, J. Llorens, E. Sacristan, J. M. Ribo, ChemPhysChem 2010, 11, 3511-3516.

[8] a) O. Arteaga, A. Canillas, R. Purrello, J. M. Ribo, Opt. Lett. 2009, 34, 2177-2179; b) O. Arteaga, C. Escudero, G. Oncins, Z. El-Hachemi, J. Llorens, J. Crusats, A. Canillas, J. M. Ribo, Chem. Asian J. 2009, 4, 1687-1696.

[9] a) J. M. Ribo, J. Crusats, F. Sagues, J. Clareet, R. Rubires, Science 2001, 292, 2063-2066; b) C. Escudero, J. Crusats, I. Diez-Perez, Z. El-Hachemi, J. M. Ribo, Angew. Chem. 2006, 118, 8200-8203; Angew. Chem. Int. Ed. 2006, 45, 8032-8035.

[10] O. Arteaga, A. Canillas, J. Crusats, Z. El-Hachemi, J. Llorens, A. Sorrenti, J. M. Ribo, Isr. J. Chem. 2011, 51, 1007-1016.

[11] P. Cheng, X. Ma, K. Hu, Y. Rong, M. Liu, Chem. Eur. J. 2011, 17, $12108-12114$.

[12] Z. El-Hachemi, C. Escudero, O. Arteaga, A. Canillas, J. Crusats, G. Mancini, R. Purrello, A. Sorrenti, A. D'Urso, J. M. Ribo, Chirality 2009, 21, 408-412.

[13] J. Crusats, J. Claret, I. Diez-Perez, Z. El-Hachemi, H. GarciaOrtega, R. Rubires, F. Sagués, JM. Ribo, Chem. Commun. 2003 $1588-1589$.

[14] e.g.; a) R. Rubires, J.-A. Farrera, J. M. Ribo, Chem. Eur. J. 2001, 7, 436-446; b) Y. Kitagawa, H. Segawa, K. Ishii, Angew. Chem. 2011, 123, 9299-9302; Angew. Chem. Int. Ed. 2011, 50, 9133-9136.

[15] Known as the Grötthus mechanisms, see, for example: a) M. Tuckerman, K. Laasonen, M. Sprik, M. Parrinello, J. Chem. Phys. 1995 103, 150-161; b) T. J. F. Day, H. W. Schmitt, G. A. Vogth, J. Am Chem. Soc. 2000, 122, 12027-12028.

[16] E. Havinga, Biochim. Biophys. Acta 1954, 13, 171-174.

[17] R. Rubires, J. Crusats, Z. El-Hachemi, T. Jaramillo, M. Lopez, E. Valls, J.-A. Farrera, J. M. Ribo, New J. Chem. 1999, 23, 189-198.

[18] a) G. E. Jellison, F. A. Modine, Appl. Opt. 1997, 36, 8184-8189; b) G. E. Jellison, F. A. Modine, Appl. Opt. 1997, 36, 8190-8198.

[19] C. Escudero, Z. El-Hachemi, J. Crusats, J. M. Ribo, J. Porphyrins Phthalocyanines 2005, 9, 852-863.

[20] a) T. P. G. Sutter, R. Rahimi, P. J. Hambright, P. J. Bommer, M. Kumar, P. Neta, J. Chem. Soc. Faraday Trans. 1993, 89, 495-502; b) J. Crusats, PhD Thesis, University of Barcelona (Spain) 1996

Received: March 15, 2012 Published online: June 8, 2012 